\title{
Near-Earth Objects 400 Years after Galileo: Physical Properties and Internal Structure
}

\author{
Dmitrij Lupishko ${ }^{1}$ and Zhanna Pozhalova ${ }^{2}$ \\ ${ }^{1}$ Institute of Astronomy of Kharkiv V. N. Karazin National University, \\ Sumska str., 35, Kharkiv 61022, Ukraine \\ email: lupishko@astron.kharkov.ua \\ ${ }^{2}$ Research Institute "Nikolaev Astronomical Observatory", \\ Observatornaya str. 1, Nikolaev 54030, Ukraine \\ email: zhanna@mao.nikolaev.ua
}

\begin{abstract}
The review contains the most recent data on near-Earth objects such as their sizes and densities, rotation and shapes, taxonomy and mineralogy, optical properties and structure of their surfaces, binary systems among the NEOs and internal structure of asteroids and comets constituted the NEO population.
\end{abstract}

Keywords. Asteroids, near-Earth objects, physical properties, internal structure.

\section{Introduction}

Near-Earth objects (NEOs) are defined as asteroids and comets having orbits with perihelion distances of $1.3 \mathrm{AU}$ or less. About $30 \%$ of the entire NEO population may reside in orbits having a Jovian Tisserand parameter $<3$, and among them roughly half are observed to have comet-like physical properties such as albedos and spectra. Thus, about $10-15 \%$ of the NEO population may be comprised by extinct or dormant comets (Lupishko \& Lupishko 2001; Binzel \& Lupishko 2006; Michel \& Bottke 2009). The rest are the near-Earth asteroids, which are traditionally divided into three groups (the relative abundances are estimated by Bottke et al. (2002)):

$$
\begin{aligned}
& \text { Amor } \quad \mathrm{a} \geqslant 1.0 \mathrm{AU} 1.017<\mathrm{q} \leqslant 1.3 \mathrm{AU} \quad(32 \pm 1 \%) \\
& \text { Apollo } \mathrm{a} \geqslant 1.0 \mathrm{AU} \quad \mathrm{q}<1.017 \mathrm{AU} \quad(62 \pm 1 \%) \\
& \text { Aten } \mathrm{a}<1.0 \mathrm{AU} \quad \mathrm{Q}>0.983 \mathrm{AU} \quad(6 \pm 1 \%)
\end{aligned}
$$

Besides, there is an additional group of rather dangerous asteroids whose orbits reside entirely inside of the Earth's one $(\mathrm{Q}<0.983 \mathrm{AU})$. According to (Michel et al. 2000) objects of this inner-Earth asteroid group and Aten group together can constitute about $20 \%$ of the km-sized Earth-crossing population. About 6730 NEOs are discovered by the beginning of 2010. They are the objects of a special interest from the point of view not only of the basic science but of the applied science as well (the problem of asteroid and comet hazard, the NEAs as the potential sources of raw materials in the nearest to the Earth space, etc.).

\section{Sizes, densities and axis rotation}

The size distribution of NEOs can be approximated as $\mathrm{N}(>\mathrm{D} \mathrm{km})=\mathrm{k} \mathrm{D}^{-b}$ with an exponent $\mathrm{b}=1.95$ and $\mathrm{k}=1090$ (Stuart 2003). This expression indicates that there are 1090 NEOs with $D \geqslant 1 \mathrm{~km}$. Including uncertainties, Stuart and Binzel (2004) give this 
result as $1090 \pm 180$ objects that are $1 \mathrm{~km}$ or lager within the NEO population. Below (Table 1) the sizes of some individual objects are presented which display the whole range of sizes of cataloged NEOs overlapping four orders of magnitude.

Table 1.

\begin{tabular}{llll}
\hline Largest NEOs & $\mathbf{D}[\mathbf{k m}]$ & Smallest discovered NEOs & $\mathbf{D}[\mathbf{m}]$ \\
\hline 1036 Ganymed & 38.5 & $\ldots$ & $\ldots$ \\
433 Eros & 16.5 & 2000 WL107 & 38 \\
3552 Don Quixote & $12 \div 15$ & 2003 QB30 & 17 \\
1866 Sisyphus & 8.9 & 2003 SQ222 & 10 \\
$\ldots$ & $\ldots$ & 2008 TC3* & 4 \\
\hline
\end{tabular}

* discovered on 6 Oct. 2008, came into collision with the Earth on 7 Oct. 2008 and disintegrated in atmosphere over northern Sudan.

The most reliable estimates of bulk densities $\left(\mathrm{g} / \mathrm{cm}^{3}\right)$ for $\mathrm{S}, \mathrm{Q}$ and C-type NEOs are summarized in Table 2. Discovery of binary NEOs gives a good opportunity to determine their bulk densities, however those estimates are usually not accurate enough due to an uncertainty of binary system parameters.

Table 2.

\begin{tabular}{lll}
\hline 433 Eros" & $2.67 \pm 0.03$ & $\mathrm{~S}$ \\
6489 Golevka* & $2.7(+0.4,-0.6)$ & $\mathrm{Q}$ \\
25143 Itokawa" & $1.95 \pm 0.14$ & $\mathrm{~S}, \mathrm{Q}$ \\
1999 KW4* & $1.97 \pm 0.24$ & $\mathrm{~S}$ \\
2100 Ra-Shalom* & $1.1-3.3$ & $\mathrm{C}$ \\
1996 FG3 & $1.4 \pm 0.3$ & $\mathrm{C}$ \\
2000 DP107 & $1.6(+1.2,-0.9)$ & $?$ \\
2000 UG11 & $1.5(+0.6,-1.3)$ & $?$ \\
\hline
\end{tabular}

Notes:

" space mission data; * radar data

Comparing bulk densities of these NEOs with those of their meteorite analogues (ordinary or carbon chondrites) we have to suppose about $30 \div 50 \%$ of the NEO porosity. It means that at least some of NEOs are not monolithic bodies but "rubble-pile" structures, which have no coherent tensile strength and weakly held together by their own mutual gravity.

The distribution of the rotation rates of NEOs (Figure 1) is quite different in comparison with that for small main-belt asteroids (MBAs) and it shows the prominent excesses of slow and fast rotators (Lupishko et al. 2007). Among the reasons for that can be the difference in asteroid diameter distributions within these two populations, influence the radiation pressure torques (YORP-effect), the influence of the rotational parameters of binaries and may be some selection effects. The whole interval of NEO rotation periods ranges over four orders of magnitudes from 500-600 hrs (96590 1998 XB and 1997 AE12) to $1.3 \mathrm{~min}$ (2000 DO8). It is clear that such small (tens meters in sizes) and super-fast rotating bodies are beyond the rotational breakup limit for aggregates like "rubble piles" and they are monolithic fragments. 


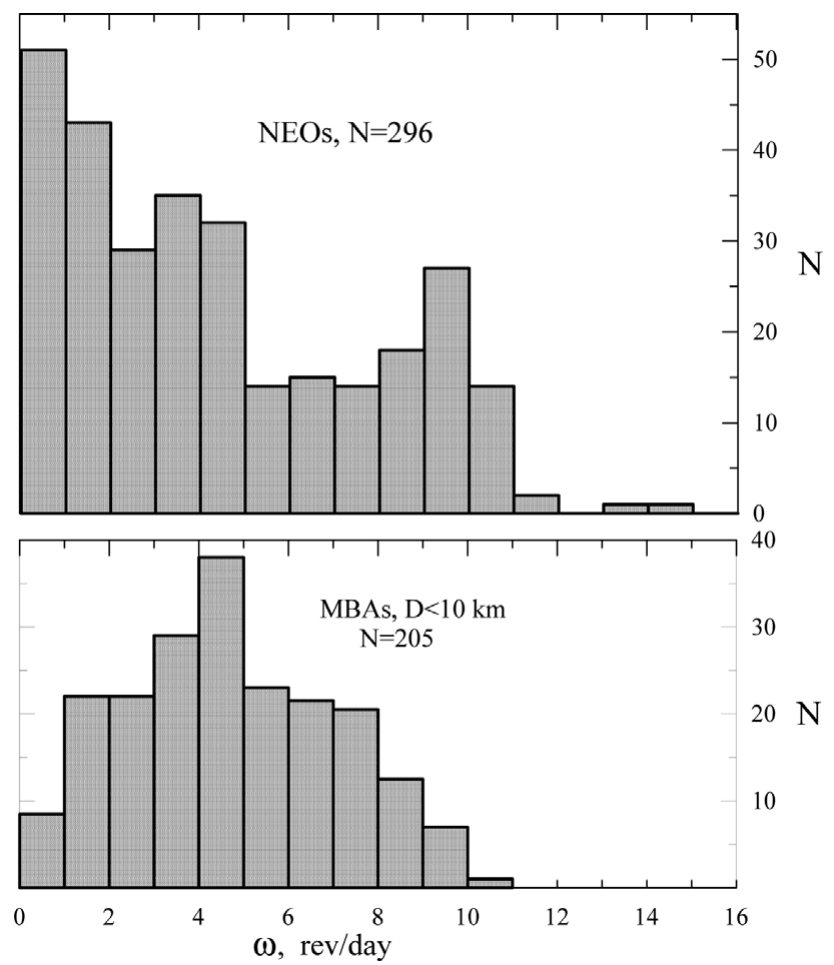

Figure 1. Distribution of the rotation rates of NEOs and small $(\mathrm{D} \leqslant 10 \mathrm{~km}) \mathrm{MBAs}$.

\section{Taxonomy and mineralogy}

As a first step toward estimating the nature of any NEO is determination of its taxonomic class, that is, the object total mineralogy. Practically all taxonomic classes identified among main-belt asteroids have been also found in NEO population, including the C, P and D classes that are typical of outer main belt. Binzel et al. (2004) from their spectroscopic survey of 252 NEAs and Mars-crossers noted that 25 of 26 Bus' taxonomic classes of MBAs are represented in the NEO-population. The most common taxonomic classes among them are however S and Q (silicate) types. Recent spectroscopic investigation of 150 NEAs (Lazzarin et al. 2008) have summarized that $62 \%$ of them belong to S-complex, $20 \%$ to X-complex, $12 \%$ to C-complex and $6 \%$ to other classes of Bus' taxonomy. Stuart and Binzel (2004) modeled the bias-corrected distribution of taxonomic classes and obtained that $\mathrm{C}$ and other low-albedo classes consist of $27 \%$ and $\mathrm{S}+\mathrm{Q}$ classes $36 \%$ of all NEOs.

Observing smaller and smaller S-objects Binzel et al. (2001) showed a continuous range of NEO spectra from those of S-types to ordinary chondrites. That is, there is a continuous transition from spectra of S-types to those of Q-types. At the same time Q-objects are smaller in sizes and brighter than S-objects, that is, their surfaces are "younger, fresher". Therefore, this continuum is interpreted as a result of space weathering process, that is, the process of alteration of the young surface of Q-asteroid to look more and more redder like S-type surface (Binzel et al. 2004). Lazzarin et al. (2008) found that only the $17 \%$ of NEOs and $6 \%$ of MBAs are compatible with ordinary chondrite spectra but other objects are much redder. They also found the statistically valid linear increase of spectral slope with increase of asteroid exposure (that is, amount of Sun's radiation that a body receives along its orbit) what support the idea of space weathering. Fevig 
and Fink (2007) reported the results of spectrophotometry of 55 NEOs which revealed the evidence for orbit-dependent trends in their data: while observed S-types reside in orbits which do not cross the asteroid main belt, the majority of objects with spectra of ordinary chondrites (Q-types, fresh and relatively unweathered NEOs) are in highly eccentric Apollo orbits which enter the asteroid main belt. Very likely that these objects have recently been injected into such orbits after a collision in the main belt.

\section{Optical properties and surface structure}

The analysis of available data clearly demonstrates that the surfaces of NEOs display in general the same optical properties as the surfaces of MBAs (Binzel et al. 2002; Lupishko \& Di Martino 1998; Lupishko et al. 2007). The whole range of NEO albedos $(0.05 \div 0.50)$ is basically the same as that of MBAs and it corresponds to the same in general mineralogy within these two populations. But the strict similarity of the other photometric and polarimetrical parameters (such as phase coefficient, polarization slope and others, which are related to surface structure) gives evidence of the similar surface structures at submicron scale.

The polarimetric, radiometric data and direct imaging of Eros and Itokawa give evidence that most of NEOs are covered with regolith (fine granulated rocks and dust). Despite their low gravities, even the smallest NEOs appear capable of retaining some regolith coating. As it was estimated, a minimum $2.3 \pm 0.4 \mathrm{~m}$ thick layer of regolith exists in the lowlands of Itokawa, which, if spread evenly across the entire asteroid, corresponds to a $42 \pm 1 \mathrm{~cm}$ layer. The recent studies of NEO thermal IR emission showed that the average thermal inertia of $\mathrm{km}$-size NEOs is $200 \pm 40 \mathrm{Jm}^{-2} \mathrm{~s}^{-0.5} \mathrm{~K}^{-1}$, that is about four times that of the Moon (Delbo et al. 2007). The authors identify also a trend of increasing thermal inertia with decreasing asteroid diameter. Radar observations showed that even the relatively small NEOs 4179 Toutatis and 1999 JM8 (D 3 km both) are cratered at about the same extent as MBAs 951 Gaspra and 243 Ida. The radar data also evidence that NEO surfaces are rougher than surfaces of large MBAs at the scale length of decimeters and meters. Recently the radar observations have also revealed a link between NEO composition and centimeter-to-decimeters surface roughness (see http://echo.jpl.nasa.gov). The most rough are the high-albedo objects of $\mathrm{E}$ and V-types, the meteorite analogs of which are enstatite chondrites and HED-meteorites (basalts), and they are more rough most probably due to higher strength of their material.

\section{Binary and triple systems among the NEOs}

By the beginning of 201037 binary near-Earth asteroids (two of them with two satellites) have been discovered. They show the similarity of their parameters, for example, rotation periods of primaries are within the interval of $2.3 \div 3.6 \mathrm{hrs}$ and orbital periods of secondaries are in the range of $0.5 \div 1.8$ days (what may be due to observational selection effects). A fraction of binary systems among the NEAs is estimated to be $15-17 \%$ (Merline et al. 2002), though among the Aten-asteroids the fraction can be significantly higher (Polishook \& Brosch 2008).

The NEA 2001 SN263 has been revealed as the first near-Earth triple asteroid ever found. It was discovered by Mitchal Nolan and his colleagues using the Arecibo radar. The central body is spherical of $\mathrm{D} \approx 2 \mathrm{~km}$ across, while the larger of the two moons is about half that size. The smallest object is about the size of the Arecibo telescope. Pravec and Harris (2007) suggest that binaries formed from parent bodies spinning at the critical rate by some sort of fission or mass shedding, and the YORP-effect is a candidate to 
be the dominant cause of spin-up to instability. This suggestion is in a good agreement with results obtained by Walsh and Richardson (2008) that tidal disruption due to close planetary encounters should account for about 1-2\% of NEAs being binaries and that there are other formation mechanisms that contribute significantly to this population. Discovery and study the binary or triple systems allow us to determine the density of the NEOs and type of their material.

\section{On the internal structure of NEOs}

There are only indirect data on the internal structure of NEOs such as bulk densities and porosities of them, their rotational rates, the events of comet nuclei disintegration, existence of large craters, crater chains and grooves on asteroids and satellites and the recent data on asteroid Itokawa. Campo Bagatin (2008) analyzed these evidences in order to extract information on the internal structure of NEOs. Taking into account the results of his analysis and other available data one can summarize that the NEO population presents at least three very different types of body internal structures. They are: a) monolithic objects (the fragments of larger parent main-belt asteroids) including the metal ones with a tensile strength of about $10^{9}$ dyne $/ \mathrm{cm}^{2}$, b) the structures of "rubblepiles" type or gravitational agregates and there are data that fraction of such bodies can be rather big (25143 Itokawa is considered as the most striking example of such structure, taking into account its density and macroporosity, an availability of large blocks on its surface and other evidences of a catastrophic disruption scenario for its formation) and c) about $10-15 \%$ of extinct or dormant comet nuclei with a tensile strength of about $10^{2}-10^{3}$ dyne $/ \mathrm{cm}^{2}$.

One can expect that forthcoming ESA space mission ISHTAR (Internal Structure High-resolution Tomography by Asteroid Rendezvous), which foresees the investigation of two Apollo-objects of different types, will supply us with new and valuable information on the internal structure of NEOs.

\section{References}

Binzel, R. P., Harris, A. W., Bus, S. J., \& Burbine, Th. H. 2001, Icarus, 151, 139

Binzel, R. P. \& Lupishko, D. F 2006, Asteroids, Comets, and Meteors, Eds. D. Lazzaro et al. Proc. of IAUS 229 “ACM 2005” Aug. 7-12, 2005. Buzios, Rio de Janeiro, Brazil. Cambridge Univ. Press, 207

Binzel, R. P., Lupishko, D. F., Di Martino, M., Whiteley, R. J., \& Hahn, G. J. 2002, Asteroids III, (W. Bottke et al., eds.) Univ. Arizona Press, Tucson, 255

Binzel, R. P., Rivkin, A. S., Scott, J. S., Harris, A. W., Bus, S. J., \& Burbine, Th. H. 2004, Icarus, 170, 259

Bottke, W. F. Jr., Morbidelli, A., Jedicke, R., Petit, J-M., Levison, H. F., Michel, P., \& Metcalfe, T. S. 2002, Icarus, 156,399

Campo Bagatin, A. 2008, Mem. S.A.It. Suppl., 12, 150

Delbo, M., Dell'Oro, A., Harris, A. W., Mottola, S., \& Mueller, M. 2007, Icarus, 190, 236

Feving, R. A. \& Fink, U. 2007, Icarus, 188, 175

Harris, A. W. \& Pravec, P. 2006, Asteroids, Comets, and Meteors., (Lazzaro D. et al., eds.) Proc. of IAUS 229, Aug. 7-12, 2005. Buzios, Rio de Janeiro, Brazil. Cambridge Univ. Press, 439

Lazzarin, M., Magrin, S., \& Marchi, S. 2008, Mem. SAI Suppl., 12, 20

Lupishko, D. F. \& Di Martino, M. 1998, Planet. Space Sci., 46, No.1, 47

Lupishko, D. F., Di Martino, M., \& Binzel, R. P. 2007, Near Earth Objects, our Celestial Neighbors: Opportunity and Risk, Proc. IAUS236, 2006 (A. Milani et al., eds.), 251

Lupishko, D. F. \& Lupishko, T. A. 2001, Solar System Research, 35, No. 3, 227 
Merline, W. J., Weidenschilling, S. J., Durda, D. D., Margot, J-L., Pravec, P., \& Storrs, A. D. 2002, Asteroids III, (W. F. Bottke et al., eds.), Univ. of Arizona Press, Tucson (USA), 289

Michel, P. \& Bottke, W. F. 2009, Book of Abstracts of Intern. Conf. "Asteroid-Comet Hazard - 2009, Russia, St. Petersburg, Sept. 21-25, 14

Michel, P., Zappala, V., Cellino, A., \& Tanga, P. 2000, Icarus, 143, 421

Polishook, D. \& Brosch, N. 2008, Icarus, 194, 111

Pravec, P. \& Harris, A. W. 2007, Icarus, 190, 250

Stuart, J. S. 2003, Ph. D. Thesis. Massach. Inst. of Technology, Cambridge, Mass.

Stuart, J. S. \& Binzel, R. P. 2004, Icarus, 170, 295

Walsh, K. J. \& Richardson, D. C. 2008, Icarus, 193, 553 